\title{
No-reflow and platelet reactivity in diabetic patients with ST-segment elevation myocardial infarction: is there a link?
}

\author{
Wiktor Kuliczkowski ${ }^{1}$, Karol Miszalski-Jamka², Jacek Kaczmarski ${ }^{3}$, Damian Pres $^{3}$, Mariusz Gąsior ${ }^{3}$ \\ ${ }^{1}$ Department and Clinic of Cardiology, Wroclaw Medical University, Wroclaw, Poland \\ ${ }^{2}$ Division of Magnetic Resonance Imaging, Silesian Center for Heart Disease, Zabrze, Poland \\ ${ }^{3} 3^{\text {rd }}$ Department of Cardiology, School of Medicine with the Division of Dentistry in Zabrze, Medical University of Silesia, Katowice, Silesian \\ Centre for Heart Disease in Zabrze, Poland
}

Adv Interv Cardiol 2017; 13, 4 (50): 326-330

DOI: https://doi.org/10.5114/aic.2017.71615

\section{Introduction}

The no-reflow phenomenon in percutaneous coronary intervention $(\mathrm{PCl})$ is defined classically as the absence of flow after restoration of arterial patency. Further research has shown that despite flow restoration in myocardial infarction ( $\mathrm{MI}$ ) there is still a considerable percentage of patients with a lack of perfusion at the level of the microvasculature caused by microvascular obstruction (MVO) [1]. This has a deleterious effect on outcomes and should be considered as a form of no-reflow [2]. It can be diagnosed with angiography or as the absence of ST-segment normalization in ECG after $\mathrm{PCl}$, but the reference method for MVO diagnosis is contrast-enhanced cardiac magnetic resonance (CMR) [3]. Causes of MVO are thought to include peripheral embolism caused by debris originating in and flushed from the atherosclerotic plaque, ischemia/reperfusion injury, and individual predispositions such as diabetes [4]. Recently, increased platelet reactivity was proposed as one of the reasons for MVO occurrence [5-7]. Increased platelet reactivity is present in diabetes and together can increase MVO. Nevertheless, there is still a considerable lack of data regarding platelet reactivity and MVO assessed with the reference method of CMR in diabetic patients with ST-segment elevation MI (STEMI).

\footnotetext{
Aim

The aim of the study was to assess the link between platelet reactivity and the occurrence of MVO in diabetic patients with STEMI.
}

\section{Material and methods}

This is a sub-study of a larger series of patients included in the previously reported data [8]. In brief the previous study included patients with STEMI treated with PCI and diagnosed with diabetes before hospital admission. To have a closer look into MVO we included patients with Thrombolysis In Myocardial Infarction (TIMI) flow grade 3 after $\mathrm{PCl}$ and defined MVO when myocardial perfusion grade (MPG) assessed with angiography was $0-1$ while normal microvascular flow was defined when MPG was 2 or 3 [1]. Study exclusion criteria were cardiogenic shock, platelet count below $100000 / \mathrm{m}^{3}$ or above $450 \mathrm{000} / \mathrm{m}^{3}$, known allergy to acetylsalicylic acid or thienopyridine derivatives, and use of GP Ilb/Illa blockers during PCl. Patients included in the study were treated according to guidelines [9]. Antiplatelet treatment included a loading dose of $600 \mathrm{mg}$ clopidogrel and a loading dose of $300 \mathrm{mg}$ aspirin given by the paramedics before hospital admission. Blood for platelet reactivity was collected into hirudin anticoagulant probes (Sarstedt, Germany) before the procedure, immediately after it and then $24 \mathrm{~h}$ after PCI. Up to $2 \mathrm{~h}$ after blood collection platelet reactivity to arachidonic acid (response to aspirin), adenosine diphosphate (ADP) (response to clopidogrel), collagen and thrombin receptor-activating peptide (TRAP) was assessed using a Multiplate impedance aggregometer (Roche, Switzerland). Agonists for platelet aggregation were obtained from the Multiplate manufacturer. High on aspirin and clopidogrel treatment platelet reactivity (HPR) were assessed according to proposed cut-offs [10]; clopidogrel HPR was diagnosed when aggregation with ADP was above

\section{Corresponding author:}

Wiktor Kuliczkowski MD, PhD, Department and Clinic of Cardiology, Wroclaw Medical University, 213 Borowska St, 50-556 Wroclaw, Poland, phone: +48 603707 093, e-mail: wkuliczkowski@wp.pl

Received: 13.08.2017, accepted: 27.09.2017. 
468 AUC $\cdot$ min and aspirin HPR was diagnosed when aggregation with arachidonic acid was above $300 \mathrm{AUC} \cdot \mathrm{min}$.

In this sub-study conducted in the year 2015 we screened 41 patients and finally included 30 patients, in whom we additionally performed contrast-enhanced CMR 7 days after PCI. Eleven drop-outs at that stage of the study were due to hospital death in 3 cases and withdrawal of informed consent in 8 patients. All CMR examinations were performed on a $1.5 \mathrm{~T}$ scanner (Magnetom Avanto, Siemens, Erlangen, Germany) with standard acquisition methods. Images were obtained during suspended respiration at end-expiration. Cine and delayed enhancement images were acquired in three standard left ventricular (LV) long-axis views and a set of multiple contiguous short-axis slices from the atrioventricular ring to the apex. Late gadolinium enhancement (LGE) imaging was performed using a segmental inversion recovery sequence $15 \mathrm{~min}$ after intravenous injection of $0.2 \mathrm{mmol} /$ $\mathrm{kg}$ gadobenate dimeglumine (Gd-BOPTA) (Multihance, Bracco, Konstanz, Germany). Images were analyzed with

Table I. Clinical characteristics of the studied group. Microvascular obstruction diagnosed according to the cardiac magnetic resonance late enhancement results

\begin{tabular}{|c|c|c|c|}
\hline Clinical variable & $\begin{array}{l}\text { Whole group } \\
\quad(n=30) \\
(1)\end{array}$ & $\begin{array}{c}\text { MVO present } \\
(n=10) \\
(2)\end{array}$ & $\begin{array}{c}\text { MVO absent } \\
(n=20) \\
(3)\end{array}$ \\
\hline Age [years] (mean \pm SD) & $59.1 \pm 9.4$ & $58.1 \pm 10.6$ & $59.5 \pm 9.2$ \\
\hline Sex (men/women) & $21 / 9$ & $6 / 4$ & $15 / 5$ \\
\hline Culprit vessel (LAD/Cx/RCA) & $10 / 5 / 15$ & $4 / 2 / 4$ & $6 / 4 / 10$ \\
\hline Number of stents used, median (min.-max.) & $1(1-3)$ & $1(1-3)$ & $1(1-2)$ \\
\hline Length of implanted stents, mean (median) [mm] & $20(18)$ & $18(18)$ & $20(18)$ \\
\hline Diameter of implanted stents, mean (median) [mm] & $3.0(3.0)$ & $3.2(3.0)$ & $3.0(3.0)$ \\
\hline Arterial hypertension, $n(\%)$ & $25(83)$ & $9(90)$ & $16(80)$ \\
\hline Hypercholesterolemia, $n(\%)$ & $24(80)$ & $8(80)$ & $16(80)$ \\
\hline Current cigarette smoking, $n(\%)$ & $10(33)$ & $4(40)$ & $6(30)$ \\
\hline Positive family history, $n(\%)$ & $4(13)$ & $1(10)$ & $3(15)$ \\
\hline Maximum troponin I level [ng/ml] $($ mean $\pm \mathrm{SD})$ & $20.1 \pm 7.9$ & $23.7 \pm 4.3^{*}$ & $19.1 \pm 3.8$ \\
\hline $\mathrm{HbA}_{1 \mathrm{c}}$ on admission (mean $\left.\pm \mathrm{SD}\right)(\%)$ & $7.7 \pm 2.5$ & $6.8 \pm 1.1$ & $7.8 \pm 2.1$ \\
\hline Left ventricular ejection fraction at discharge $($ mean \pm SD) $(\%)$ & $45.2 \pm 4.6$ & $43.1 \pm 4.0^{*}$ & $47.2 \pm 3.4$ \\
\hline Statins, $n(\%)$ & $30(100)$ & $10(100)$ & $20(100)$ \\
\hline$\beta$-Blockers, $n(\%)$ & $22(73)$ & $8(80)$ & $14(46)$ \\
\hline ACE-I, $n(\%)$ & $30(100)$ & $10(100)$ & $20(100)$ \\
\hline Aspirin $1 \times 75 \mathrm{mg}, n(\%)$ & $30(100)$ & $10(100)$ & $20(100)$ \\
\hline Clopidogrel $1 \times 75$ mg, $n(\%)$ & $30(100)$ & $10(100)$ & $20(100)$ \\
\hline \multicolumn{4}{|l|}{ Diabetes treatment at hospital admission, $n(\%)$ : } \\
\hline Insulin & $7(23)$ & $2(20)$ & $5(25)$ \\
\hline Biguanides & $10(33)$ & $5(50)$ & $5(25)$ \\
\hline Sulphonylurea derivatives & $13(43)$ & $2(20)$ & $11(55)$ \\
\hline \multicolumn{4}{|l|}{ Diabetes treatment during acute STEMI phase, $n(\%)$ : } \\
\hline Insulin & $15(50)$ & $5(50)$ & $10(50)$ \\
\hline Biguanides & $14(47)$ & $5(50)$ & $9(45)$ \\
\hline Sulphonylurea derivatives & $1(3)$ & $0(0)$ & $1(5)$ \\
\hline
\end{tabular}

${ }^{*} p<0.05$ (2) vs. (3). MVO - microvascular obstruction, LAD - left anterior descending artery, Cx - circumflex artery, RCA - right coronary artery, ACE-I - angioten sin-converting-enzyme inhibitor, $H b A_{1 c}$ - glycated hemoglobin, SD - standard deviation. 
commercial software (Qmass MR, Medis Medical Imaging Systems, Leiden, the Netherlands). Left ventricular volumes and ejection fraction were calculated on the basis of end-diastolic and end-systolic manual endocardial tracings. Presence of MVO on LGE images was considered as the CMR criterion for no-reflow. Specifically, MVO was defined as a hypointense region surrounded by a hyperintense area (i.e. enhanced myocardium) on LGE images. Infarct size was quantified using computer-assisted planimetry and reported as percent of LV mass.

The study was approved by the Bioethics Committee of the Medical University of Silesia. All patients were required to sign an informed consent form for study participation.

\section{Statistical analysis}

Data are presented as mean \pm SD for the normally distributed parameters. Depending on data distribution
Student's $t$-test or Mann-Whitney $U$ test was employed for comparison of 2 groups. For categorical or nominal variables the $\chi^{2}$ test with adjustment for small numbers where applicable was used. Statistica 12 software (StatSoft, USA) was used for all calculations.

\section{Results}

Clinical characteristics of the study population are shown in Table I. Door-to-balloon time in the study was $74 \pm 19$ min. Mean \pm SD time from aspirin and clopidogrel loading dose and $\mathrm{PCl}$ was $57 \pm 17 \mathrm{~min}$. The CMR defined MVO was present in 10 out of 30 patients. We found that platelet reactivity measured by four agonists was significantly higher in the group with MVO before and after the procedure, but not after $24 \mathrm{~h}$ (Table II). The HPR on aspirin was present in 10 patients before $\mathrm{PCl}$ and in 9 after $\mathrm{PCl}$ and it was more frequent in the MVO group in comparison with good myocardial flow on CMR (50\% vs. $25 \%$

Table II. Platelet reactivity and left ventricle performance according to microvascular obstruction diagnosed with cardiac magnetic resonance late enhancement

\begin{tabular}{|c|c|c|c|}
\hline Variable & $\begin{array}{c}\text { CMR LE } \\
\text { MVO present } \\
(n=10)\end{array}$ & $\begin{array}{c}\text { CMR LE } \\
\text { MVO absent } \\
(n=20)\end{array}$ & $\begin{array}{l}\text { Statistical } \\
\text { significance }\end{array}$ \\
\hline \multicolumn{4}{|l|}{ Before PCl: } \\
\hline AA induced aggregation (AUC, AU $\cdot \min )($ mean $\pm S D)$ & $398 \pm 30$ & $214 \pm 17$ & $p<0.04$ \\
\hline ADP induced aggregation (AUC, AU $\cdot \min )($ mean $\pm S D)$ & $1179 \pm 270$ & $685 \pm 399$ & $p<0.01$ \\
\hline TRAP induced aggregation (AUC, AU $\cdot$ min) (mean \pm SD) & $1426 \pm 443$ & $1099 \pm 171$ & $p<0.04$ \\
\hline COL induced aggregation (AUC, AU $\cdot \min )($ mean $\pm S D)$ & $829 \pm 44$ & $398 \pm 21$ & $p<0.001$ \\
\hline$\overline{A A}$ induced aggregation (AUC, $A U \cdot \min )($ mean $\pm S D)$ & $317 \pm 18$ & $181 \pm 19$ & $p<0.04$ \\
\hline \multicolumn{4}{|l|}{ After PCl: } \\
\hline ADP induced aggregation (AUC, AU $\cdot \min )($ mean $\pm S D)$ & $1015 \pm 379$ & $592 \pm 356$ & $p<0.01$ \\
\hline TRAP induced aggregation (AUC, AU $\cdot \min )($ mean \pm SD) & $1376 \pm 278$ & $943 \pm 439$ & $p<0.02$ \\
\hline$\overline{C O L}$ induced aggregation $(A \cup C, A U \cdot \min )($ mean $\pm S D)$ & $815 \pm 35$ & $493 \pm 29$ & $p<0.02$ \\
\hline \multicolumn{4}{|l|}{$24 \mathrm{~h}$ after $\mathrm{PCl}$} \\
\hline AA induced aggregation (AUC, AU $\cdot \min )($ mean $\pm S D)$ & $186 \pm 17$ & $158 \pm 20$ & NS \\
\hline ADP induced aggregation (AUC, AU $\cdot \min )($ mean $\pm S D)$ & $309 \pm 33$ & $301 \pm 24$ & NS \\
\hline TRAP induced aggregation (AUC, AU $\cdot \min )($ mean \pm SD) & $811 \pm 79$ & $682 \pm 110$ & NS \\
\hline$\overline{C O L}$ induced aggregation $(A \cup C, A U \cdot \min )($ mean $\pm S D)$ & $301 \pm 82$ & $314 \pm 98$ & NS \\
\hline \multicolumn{4}{|l|}{ CMR determined LV parameters: } \\
\hline$\overline{\operatorname{LVEF}(\%)(\text { mean } \pm S D)}$ & $38.1 \pm 9.5$ & $53.1 \pm 8.9$ & $p<0.001$ \\
\hline LVEDV $[\mathrm{ml}]($ mean \pm SD) & $208.4 \pm 60.8$ & $179.7 \pm 51.2$ & NS \\
\hline$\overline{\operatorname{LVESV}[\mathrm{ml}](\text { mean } \pm \mathrm{SD})}$ & $133.1 \pm 55.3$ & $86.2 \pm 38.6$ & $p<0.05$ \\
\hline Infarct size (\% LV mass) (mean \pm SD) & $31.3 \pm 14.8$ & $12.4 \pm 7.3$ & $p<0.0001$ \\
\hline
\end{tabular}

$C M R L E$ - cardiac magnetic resonance late enhancement, MVO - microvascular obstruction, $P C I$ - percutaneous coronary intervention, $L V$ - left ventricle, $L V E F-l e f t$ ventricle ejection fraction, LVEDV - left ventricle end diastolic volume, LVESV - left ventricle end systolic volume, AA - arachidonic acid, ADP - adenosine diphosphate, TRAP - thrombin receptor activating peptide, $C O L$ - collagen, AUC - area under curve, AU - min - arbitrary units per minute, SD - standard deviation. 
before $\mathrm{PCl}$ and $50 \%$ vs. $20 \%$ after $\mathrm{PCl} ; p<0.05$ for both). HPR on clopidogrel was present in 24 patients before $\mathrm{PCl}$ and in 20 patients after $\mathrm{PCl}$ and it was more frequent in the MVO group in comparison with good myocardial flow on CMR ( $90 \%$ vs. $75 \%$ before PCl; $p<0.05)$. MVO was associated with significantly lower left ventricle ejection fraction, higher left ventricle end-systolic volume and larger infarct size. There was no significant difference between the two groups for time from antiplatelet treatment start to $\mathrm{PCl}$ (MVO present vs. MVO absent $58 \pm 11$ vs. $55 \pm 14 \mathrm{~min}$ ) nor door-to-balloon time (MVO present vs. MVO absent $77 \pm 22$ vs. $73 \pm 19 \mathrm{~min}$ ). There was a significant positive correlation between CMR defined infarct size (\% of the left ventricle mass) and platelet reactivity before PCI to arachidonic acid $(r=0.46)$, ADP $(r=0.55)$ and collagen $(r=0.46$; all $p<0.05)$.

\section{Discussion}

The impact of platelets on MVO presence was investigated previously by our group and others [7, 8, 11-13]. Interestingly, there are few studies comparing platelet reactivity in STEMI with MVO diagnosed with CMR, which seems to be a "gold standard" of MVO assessment [3]. We found that four different platelet activation pathways were highly reactive in vitro in MVO in comparison with good microvascular flow. This result adds to existing evidence; not only poor response to aspirin and clopidogrel was associated with more frequent MVO, but also increased platelet activation pathway through collagen and thrombin receptor. In other words, obtaining TIMI 3 flow in primary $\mathrm{PCl}$ does not guarantee good microvascular flow on CMR if platelets show higher reactivity in four different pathways. This association disappeared $24 \mathrm{~h}$ after $\mathrm{PCl}$, which could only be attributed to the periprocedural phenomenon in patients with a good angiographic result according to TIMI flow. Zalewski et al. found that presence of MVO on CMR is associated with higher admission measured ADP induced reactivity of platelets (response to clopidogrel) but not arachidonic acid (response to aspirin). The difference for ADP reactivity was also present 4 days after $\mathrm{PCl}$ [7]. In their study $23 \%$ of patients had post-PCI TIMI less than 3 and different aggregometry than in our group, so although some results are concordant with ours they are difficult to compare. Others also showed that higher platelet reactivity assessed indirectly by means of platelet volume [11] or platelet microparticle levels [14] were all associated with presence of MVO on CMR, while lower reactivity to TRAP was associated with more frequent intramyocardial hemorrhage on CMR in patients with STEMI treated with primary $\mathrm{PCI}$ [15].

Main study limitation is the small number of patients included although we performed this subanalysis as hypothesis generating only. Strikingly, even with such small numbers the CMR defined MVO group showed a highly significant difference in periprocedural platelet reactivi- ty, which also correlated with infarct size as assessed on CMR. As CMR is a "gold standard" for the diagnosis of MVO we did not investigate MVO based on ECG or contrast echocardiography.

\section{Conclusions}

Our study indicates that periprocedural higher platelet reactivity to arachidonic acid, ADP, TRAP and collagen may be associated with presence of MVO diagnosed with CMR in diabetic patients with STEMI treated with primary $\mathrm{PCI}$.

\section{Conflict of interest}

The authors declare no conflict of interest.

\section{References}

1. Gibson CM, Cannon CP, Murphy SA, et al. Relationship of TIMI myocardial perfusion grade to mortality after administration of thrombolytic drugs. Circulation 2000; 101: 125-30.

2. Bethke A, Halvorsen S, Bohmer E, et al. Myocardial perfusion grade predicts final infarct size and left ventricular function in patients with ST-elevation myocardial infarction treated with a pharmaco-invasive strategy (thrombolysis and early angioplasty). Eurolntervention 2015; 11: 518-24.

3. Abbas A, Matthews GH, Brown IW, et al. Cardiac MR assessment of microvascular obstruction. Br J Radiol 2015; 88: 20140470.

4. Niccoli G, Scalone G, Lerman A, Crea F. Coronary microvascular obstruction in acute myocardial infarction. Eur Heart J 2016; 37 : 1024-33.

5. Cakici M, Cetin M, Balli M, et al. Predictors of thrombus burden and no-reflow of infarct-related artery in patients with ST-segment elevation myocardial infarction: importance of platelet indices. Blood Coagulation Fibrinol 2014; 25: 709-15.

6. Capranzano P, Capodanno D, Bucciarelli-Ducci C, et al. Impact of residual platelet reactivity on reperfusion in patients with ST-segment elevation myocardial infarction undergoing primary percutaneous coronary intervention. Eur Heart J Acute Cardiovasc Care 2016; 5: 475-86.

7. Zalewski J, Durak M, Lech P, et al. Platelet activation and microvascular injury in patients with ST-segment elevation myocardial infarction. Kardiol Pol 2012; 70: 677-84.

8. Kuliczkowski W, Gasior M, Pres D, et al. Aspirin 'resistance': impact on no-reflow, platelet and inflammatory biomarkers in diabetics after ST-segment elevation myocardial infarction. Cardiology 2015; 131: 41-50.

9. Task Force on the management of STEMI, Steg PG, James SK, Atar D, et al. ESC Guidelines for the management of acute myocardial infarction in patients presenting with ST-segment elevation. Eur Heart J 2012; 33: 2569-619.

10. Aradi D, Collet JP, Mair J, et al. Platelet function testing in acute cardiac care - is there a role for prediction or prevention of stent thrombosis and bleeding? Thrombosis Haemostasis 2015; 113 : 221-30.

11. Fabregat-Andres O, Cubillos A, Ferrando-Beltran M, et al. Mean platelet volume is associated with infarct size and microvascular obstruction estimated by cardiac magnetic resonance in ST segment elevation myocardial infarction. Blood Coagulation Fibrinol 2013; 24: 424-7.

12. Niccoli G, Giubilato S, Russo E, et al. Plasma levels of thromboxane $A 2$ on admission are associated with no-reflow after 
primary percutaneous coronary intervention. Eur Heart J 2008; 29: 1843-50.

13. Toprak C, Tabakci MM, Simsek Z, et al. Platelet/lymphocyte ratio was associated with impaired myocardial perfusion and both in-hospital and long-term adverse outcome in patients with ST-segment elevation acute myocardial infarction undergoing primary coronary intervention. Postep Kardiol Interw 2015; 11: 288-97.

14. Jung C, Sorensson P, Saleh N, et al. Circulating endothelial and platelet derived microparticles reflect the size of myocardium at risk in patients with ST-elevation myocardial infarction. Atherosclerosis 2012; 221: 226-31.

15. Malek LA, Klopotowski M, Spiewak M, et al. Platelet reactivity and intramyocardial hemorrhage in patients with ST-segment elevation myocardial infarction. Clin Appl Thrombosis Hemostasis 2014; 20: 553-8. 